Journal of the Magnetics Society of Japan Vol. 15 Supplement, No. S2 (1991)

(C) 1991 by The Magnetics Society of Japan

\title{
COMPARISONS OF MEASURED AND CALCULATED ANGULAR DEPENDENCE OF COERCIVITY OF LONGITUDINAL CoCr/Cr THIN FILMS
}

\author{
Mingjyue HUANG and Jack H. JUDY \\ The Center For Micromagnetics and Information Technologies (MINT) \\ Department of Electrical Engineering, University of Minnesota \\ Minneapolis, Minnesota 55455, USA
}

\begin{abstract}
With corrections for demagnetization fields in the direction of the applied external field, the effects of the easy axis distributions on the angular dependence of apparent coercivity for coherent rotation and incoherent curling and fanning rotations in longitudinal magnetic thin films are taken into account. for the thin film. Calculations are compared with the measurements for $\mathrm{CoCr} / \mathrm{Cr}$ thin films by $\mathrm{Lu}$ et al [2]. It is shown that the curves of the angular dependence of apparent coercivity for $4000 \mathrm{~A} \mathrm{CoCr} / \mathrm{Cr}$ thick films with or without $3000 \mathrm{~A} \mathrm{Cr}$ underlayer can be explained by the curling rotation process, and that a $3000 \mathrm{~A}$ thick $\mathrm{Cr}$ underlayer induces a change of the micromagnetization reversal for $350 \mathrm{~A} \mathrm{CoCr}$ thin films from fanning to curling and an increase of the in-plane component of the easy axis distributions. It is also shown that all curves of the angular dependence of the apparent coercivity for $\mathrm{CoCr} / \mathrm{Cr}$ films can be explained by components of effective in-plane isotropic and vertical-plane Gaussian easy axis distributions.
\end{abstract}

\section{INTRODUCTION}

Magnetic thin films of $\mathrm{CoCr}$ alloy on $\mathrm{Cr}$ underlayers have been reported to have great potential use as high-coercivity and low-noise longitudinal magnetic recording media [1]. The angular dependences of the apparent coercivity of $\mathrm{CoCr} / \mathrm{Cr}$ thin films have been measured and the micromagnetization reversal processes have been discussed without corrections of demagnetization fields [2]. The effects of demagnetization fields must be taken into account when comparing magnetization measurements with calculations in order to determine the most likely mechanism of magnetization reversal [3-6]. In addition, a $\mathrm{CoCr}$ alloy magnetic film deposited on a $\mathrm{Cr}$ underlayer may have a different shape of the angular dependence of coercivity due to a change in the distribution of easy axes. The easy axis distribution of the particles in thin magnetic films can have a significant effect on the angular dependence of apparent coercivity [7].

The purpose of this paper is to report on investigations of the micromagnetization reversals possible in longitudinal $\mathrm{CoCr} / \mathrm{Cr}$ thin films by comparing calculations of the angular dependence of apparent coercivity with measurements reported by $\mathrm{Lu}$ et al [2] taking into account the corrections for demagnetization fields in the direction of applied fixed-direction external field and the effects of the distribution of easy axes. This paper does not take into account the interparticle interactions and does not address the full correctons for demagnetization fields in thin films as was done experimentally by Bernards and Cramer [8] for measuring the true coercivity.

\section{CALCULATIONS}

As shown in Fig. 1, the magnetization vector, $\mathbf{M}$, of a particle in a thin film is directed at an angle $\theta$ with respect to the easy axis of the particle and rotates towards the effective field $\left(\mathrm{H}_{\mathrm{e}}\right)$ which is the summation of the demagnetization field $\left(\mathrm{H}_{d}\right)$ and the applied external field $\left(\mathrm{H}_{\mathrm{a}}\right)$ which is directed at a fixed angle of $\Omega$ in respect to the thin film. The apparent coercivity [7] is calculated as the magnitude of the fixed-direction external field when $\mathrm{M}_{\mathrm{x}}=0$. When the fixed-direction external field is applied, both the magnitude and the direction $(\alpha)$ of the effective field change. 


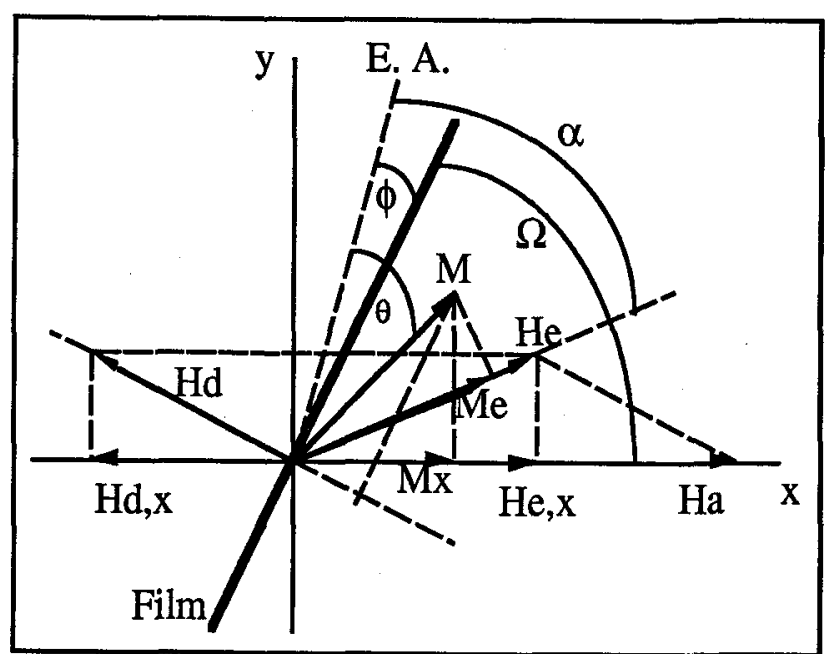

\section{Fig. 1 Geometrical relations for corrections of the demagnetization fields.}

The in-plane and vertical-plane distributions of the easy axes with 2-dimensionally Gaussian [3] and isotropic [7] shapes are considered for the calculations of the angular dependence of apparent coercivity for coherent rotation and incoherent curling and fanning rotations. The in-plane easy axis of a particle can have a value of $\phi=0$ as shown in Fig. 1. A vertical-plane easy axis distribution is expressed in the region of $-\pi / 2 \leq \phi \leq \pi / 2$. A Gaussian shape of easy axis distribution gives a spatial differential probability at an angle $\gamma$ in the following equation,

$$
\mathrm{dP} \equiv(\beta / \pi)^{1 / 2} \exp \left[-\beta\left(\gamma-\gamma_{\mathrm{p}}\right)^{2}\right] \mathrm{d} \theta,
$$

where $\gamma_{p}$ is the location of the peak magnitude of the Gaussian shape and $\beta$ is the Gaussian sharpness factor. The peak of a vertical-plane Gaussian easy axis distribution is assumed to be located in the thin film plane.

The demagnetization factor is assumed to be $4 \pi$ when the applied field is directed normal to the thin film plane and zero when the field is directed in the plane of the film. The magnitude of the demagnetization field, $\mathrm{H}_{\mathrm{d}}$, is calculated by using

$$
\mathrm{H}_{\mathrm{d}}=-4 \pi \sin \xi,
$$

where $\xi$ is the out-of-plane angle of the magnetization vector, e.g., $\xi=\theta-\phi$ for the vertical-plane easy axis distribution. The magnitude of $\mathrm{H}_{d}$ is corrected by the component of $\mathrm{H}_{\mathrm{d}, \mathrm{x}}$.
The anisotropy field, $\mathrm{H}_{\mathrm{k}}$, of a thin film single particle is assumed to be 5600 oersteds [3] for the calculations. When an external field is applied to the films, the magnetization vectors of the particles in thin film are assumed to rotate with a fixed magnitude of a saturation magnetization, $M_{s}$, of 600 and $650 \mathrm{emu} / \mathrm{cc}$ for $\mathrm{CoCr}$ films with and without $\mathrm{Cr}$ underlayer, respectively [9].

\section{RESULTS}

The measurements of the angular dependence of apparent coercivity for $\mathrm{CoCr} / \mathrm{Cr}$ films reported by $\mathrm{Lu}$ et al [2] are normalized by the zero-degree coercivity, $\mathrm{H}_{\mathrm{co}}$, and are shown as specific points in Fig. 2. The data points $\mathrm{B}$ and $\mathrm{C}$ correspond to $\mathrm{CoCr}$ films deposited without, and $\mathrm{F}$ and $\mathrm{G}$ with a 3000A Cr underlayer. Data $\mathrm{B}$ and $\mathrm{F}$ are for measurements with $350 \mathrm{~A}$ thin $\mathrm{CoCr}$ films and $\mathrm{C}$ and $\mathrm{G}$ are for $4000 \mathrm{~A}$ thick $\mathrm{CoCr}$ films. The calculated curve for B shows a fanning rotation process with a value of 2 for the number of spheres in chain (n) [10] with effectively $100 \%$ of vertical-plane Gaussian easy-axis distribution. The Gaussian sharpness

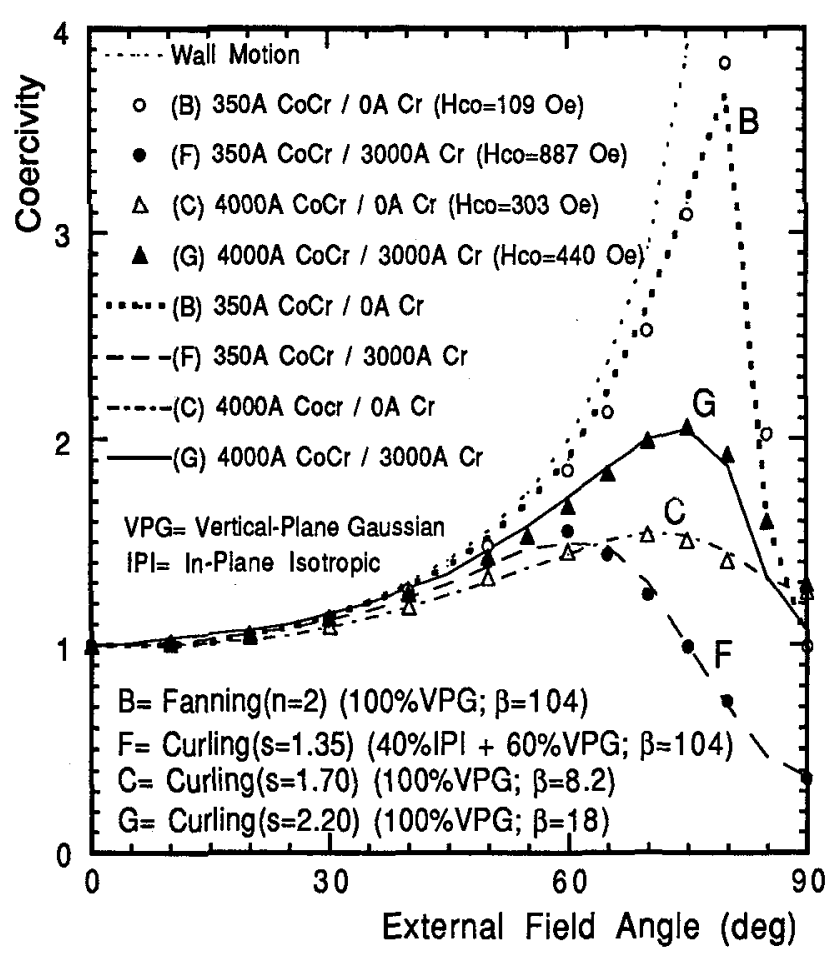

Fig. 2 Angular dependence of coercivity for $\mathrm{CoCr} / \mathrm{Cr}$ films. 
factor, $\beta$, is 104. Calculated curves for $G, C$, and $F$ data show the curling rotation processes with $2.20,1.70$, and 1.35 , respectively, of the diameter, $\mathrm{s}$, of particles, and with values of $18,8.2$, and 104 , respectively, for the Gaussian sharpeness factor. Calculated curves for $G$ and $C$ have effectively a $100 \%$ vertical-plane Gaussian easy-axis distribution. However, the calculated curve for $F$ is best represented by $40 \%$ in-plane isotropic and $60 \%$ vertical-plane Gaussian easy-axis distributions.

\section{DISCUSSION}

All curves of the measured angular dependence of apparent coercivity shown in Fig. 2 exhibit sharp peaks suggesting that the magnetization reversals are not due to a coherent rotation process [6] or wall motion. Since the peak normalized coercivity of B does not occur at an angle $>80$ degrees and is smaller than 4 , the B measurements can either be due to fanning or curling. A curling rotation with $s \geq 3.5$ may have a similar curve to $B$, but a CoCr film of $350 \mathrm{~A}$ thickness is not likely to have such a large diameter of $\mathrm{CoCr}$ particles with inplane easy axes. Accordingly, the most probable rotation process for $B$ is fanning with a minimum value of two spheres in a chain $(n=2)$. On the other hand, the ratio of zero-degree coercivities of Curves $\mathrm{F}$ and $\mathrm{B}$ is 8.13 [2], which is much greater than the ratio of the fanning constant [10], $\mathrm{K}_{\mathrm{n}}$, for $\mathrm{n}=\infty$ and $\mathrm{n}=2$, which are 1.1857 and 0.5000 respectively. This implies that the Curve $\mathrm{F}$ can not be due to any fanning rotation process if Curve $B$ is due to a fanning reversal. The $F$ reversal process is therefore probably associated with curling.

Note that a $3000 \mathrm{~A}$ thick $\mathrm{Cr}$ underlayer results in a change of micromagnetization rotation process for a $350 \mathrm{~A} \mathrm{CoCr}$ film from fanning to curling and an increase of the percentage of the in-plane easyaxis distribution. This is also the reason why the component of the effective in-plane easy axes of $G$ must be more than those in $C$. The resultant calculations indicate that Curves $\mathrm{G}$ and $\mathrm{C}$ fit a curling process which has effectively only a vertical-plane Gaussian easy-axis distribution with different values of sharpness, $\beta$. The component of the effective in-plane easy axes of $C$ is less than those in $\mathrm{G}$ due to a broader Gaussian distribution of easy axes of $\mathrm{C}$.
Based on the resultant calculations, it is noted that the $\mathrm{CoCr}$ films without $\mathrm{Cr}$ underlayer have effectively a very sharp vertical-plane Gaussian distribution of easy axes in 350A CoCr longitudinal recording thin film and a broader vertical-plane Gaussian distribution of easy axes in $4000 \mathrm{~A} \mathrm{CoCr}$ longitudinal recording thin film. Note also that the particles in $4000 \mathrm{~A} \mathrm{CoCr}$ films reversing by curling are much larger than those in a $350 \mathrm{~A}$ film.

\section{CONCLUSION}

(1) Curves of the angular dependence of the apparent coercivity for $\mathrm{CoCr} / \mathrm{Cr}$ thin films can be explained by components of effective in-plane isotropic and vertical-plane Gaussian-shaped distributions of easy axes.

(2) The magnetization of a $350 \mathrm{~A}$ thin $\mathrm{CoCr}$ film with and without a $\mathrm{Cr}$ underlayer rotates by curling and fanning, respectively.

(3) A $4000 \mathrm{~A}$ thick $\mathrm{CoCr}$ film with or without $\mathrm{Cr}$ underlayer reverses only by curling with larger diameter particles than those in the $350 \mathrm{~A}$ thin film.

(4) A 3000A thickness $\mathrm{Cr}$ underlayer induces a change of micromagnetization reversal for $350 \mathrm{~A}$ $\mathrm{CoCr}$ film from fanning to curling and an increase of component of in-plane easy-axis distribution for particles in thin film.

(5) A 4000A CoCr film with or without a $\mathrm{Cr}$ underlayer appears to reverse by curling with an effective $100 \%$ vertical-plane Gaussian distribution of easy axes.

\section{REFERENCES}

[1] Fisher, R. D., Allan, J. C., and Pressesky, J. L., IEEE Trans. Magn., vol.22, No.5, pp.352-354 (1986).

[2] Lu, M., Chen, Q., Judy, J. H., and Sivertsen, J. M., Journ. Magn. Soc., Japan, vol.13, Supplement, No.S1, pp.435-439 (1989).

[3] Nakamura, Y., and Iwasaki, S., IEEE Trans. Magn., vol.23, No.1, pp.153-155 (1987).

[4] Ouchi, K., and Iwasaki, S., IEEE Trans. Magn., vol.24, No.6, pp.3009-3011 (1988).

[5] Speliotis, D., Bono, D., and Judge, P., Journ. Magn. Soc., Japan, vol.13, Supplement, No.S1, pp.887-892 (1989). 
[6] Huang, M., and Judy, J. H., The 5th Joint MMMIntermag Conference in Pittsburgh, USA. To appear in IEEE Trans. on Magn., November (1991).

[7] Huang, M., and Judy, J. H., International Conference on Magnetism in Edinburgh, England. To be published (1991).

[8] Bernards, J. P. C., and Cramer, H. A. J., The 5th Joint MMM-Intermag Conference in Pittsburgh, USA (Paper \#CE-15) (1991).

[9] Lu, M., Private communication.

[10] Jacobs, I. S., and Bean, C. P., Phys. Rev., 100, pp.1060-1067 (1955). 